\title{
NUCLEUS ACCUMBENS: BEHAVIORAL AND COGNITIVE FUNCTIONS
}

\section{Preface}

An important issue in the field of the neurobiology of learning and memory is how associative functions of limbic and frontal cortical systems ultimately come to influence the behavior of animals. In this special issue of Psychobiology, we focus on the possibility that the nucleus accumbens (NA) plays a pivotal role in determining the behavioral consequences of limbic and neocortical mnemonic processing. Specifically, this special issue was organized to highlight the importance of the NA in mediating cognitive and behavioral processes associated with reward, learning, memory, strategy and response selection, feeding, behavioral sensitization, and drug addiction.

The first three articles represent a summary of the anatomical, neurochemical, synaptic, electrophysiological characterization of the NA, as well as a summary of the critical input and output systems. In the first article, "Hippocampal and Amygdaloid Interactions in the Nucleus Accumbens," Groenewegen et al. provide an exposition of the afferent and efferent fiber connections with the NA, especially between the hippocampus and amygdala; a discussion of the differences between the shell and core regions of the NA; and a discussion of the physiological changes induced by amygdala and hippocampal inputs and their interactions. Finally, Groenewegen et al. discuss the implications of the amygdala-hippocampal interactions. In the second article, "Microcircuits in Nucleus Accumbens' Shell and Core Involved in Cognition and Reward," Meredith and Totterdell give an excellent presentation of the neurochemical architecture, the morphological substrate, and the synaptic framework within the core and shell of the NA, followed by a discussion of how microcircuits within the NA mediate stress, detection of novelty, learning, reward, prepulse inhibition, sensory-motor function, behavioral sensitization, and drug addiction. In the third article, "Ensemble Coding in the Nucleus Accumbens," O'Donnell discusses the electrophysiological characteristics of NA medium-sized spiny neurons as providing for a bistable state that can be influenced by hippocampal, prefrontal cortex, and dopamine inputs. These in turn affect various output systems for selection of appropriate responses and for feedback onto the prefrontal cortex.

The next four articles represent an integrative view of the various functions of the NA with respect to a subregional analysis of NA function, analysis of the importance of dopaminergic innervation, or analysis of NA function within the larger striatal system. In the first article, "Neural Integrative Activities of Nucleus Accumbens Subregions in Relation to Learning and Motivation," Kelley argues that the core region of NA is involved in response-reinforcement learning, whereas the shell region of the NA is not involved in learning, but rather integrates basic drives and visceroendocrine effector system, such as eating. In the second article, "Function of the Nucleus Accumbens Within the Context of the Larger Striatal System," Mizumori, Pratt, and Ragozzino propose that the NA in conjunction with the caudate-putamen provide a mechanism for a response reference system, with the NA responding to the current situation and the caudate-putamen evaluating the current situation in the context of past experience. In the third article, "Functions of the Dopaminergic Innervation of the Nucleus Accumbens," Gray, Kumari, Lawrence, and Young suggest that the NA operates not only via its mesolimbic dopaminergic projection, but also via outputs to perceptual as well as motor systems. This suggestion provides for a vehicle to explain the behavioral effects of both reward and disruption in cognitive function that are seen in acute schizophrenia. In the fourth article, "Nucleus Accumbens Dopamine and Rate of Responding: Neurochemical and Behavioral Studies," Salamone, Aberman, Sokolowski, and Cousins make the case that dopaminergic innervation is not critical for reward, but that it does play a role in mediating motor and motivational processes.

The next four articles represent empirical findings from attempts to differentiate the contribution of the core and shell regions of NA, differentiate the contribution of the NA and caudateputamen, and integrate the dopaminergic and hippocampal inputs into NA. In the first article, "Differential Effects of Immediate Posttraining Sulpiride Microinfusions Into the Nucleus Accumbens 
Shell and Core on Morris Water Maze Retention," Setlow and McGaugh argue on the basis of manipulation of the D2 receptor that the shell and core regions of the NA may be involved in consolidation of memory for different aspects of water maze learning. In the second article, by Parkinson, Robbins, and Everitt, entitled "Selective Excitotoxic Lesions of the Nucleus Accumbens Core and Shell Differentially Affect Aversive Pavlovian Conditioning to Discrete and Contextual Cues," lesions of the shell of the NA had no deleterious effects. In the third article, by Eagle et al., entitled "Differential Effects of Ventral and Regional Dorsal Striatal Lesions on Sucrose Drinking and Positive and Negative Contrast in Rats," it is determined that NA lesions shifted the intake-concentration function to the left but did not affect positive or negative contrast. By comparison, lateral caudate-putamen lesions reduced volume consumption perhaps due to motor impairments, with no effect on positive or negative contrast. In the fourth article, "Dopamine and Hippocampal Input to the Nucleus Accumbens Play an Essential Role in the Search for Food in an Unpredictable Environment," Floresco and Phillips demonstrate that predicting the presence of food in a complex environment is mediated both by $\mathrm{Dl}$ receptors and ventral CA1/subiculum components of the hippocampus.

The next two articles elaborate on the issue of the role of the NA in behavioral sensitization. In the first one, "Neural Substrates Mediating Context-Dependent Sensitization to Psychostimulants," Nakamura, Bell, Cornish, and Kalivas review findings that suggest that context-dependent sensitization of locomotor activity may be mediated by glutamate transmission from prefrontal cortex and/or amygdala. In the second, "Environmental Enrichment Enhances the Stimulant Effect of Intravenous Amphetamine: Search for a Cellular Mechanism in the Nucleus Accumbens," Bardo, Valone, Robinet, Shaw, and Dwoskin suggest that dopamine transporter proteins in the NA may play a role in aiding environmental influences on behavioral responses to amphetamine. In the last article, "Cognitive Factors in Addiction and Nucleus Accumbens Function: Some Hints From Rodent Models," Wise reviews the role of the NA in drug addiction in both animals and humans as it is influenced by cognitive and noncognitive factors.

From these articles, it appears that there is a growing consensus that the NA integrates reward value with spatial and temporal contextual information and strategies in order to update response selection by the cortex. This is accomplished by considering inputs from the amygdala, hippocampus, and prefrontal cortex. The resultant efferent messages of the ventral pallidum are sent to the thalamus and finally the prefrontal cortex. The dopaminergic input from the ventral tegmental area is not directly involved in reward; rather, it represents a gain mechanism on the response system. It is hoped that the publication of this special issue will engender further interest in the role of the nucleus accumbens in mediating behavior and cognitive functions.

—Raymond P. Kesner and Sheri J. Y. Mizumori Special Issue Editors 